universe at critical density with the astronomers' Universe. Even the distribution of fluctuations predicted by the inflationary theory is, according to Blumenthal et al., reflected in the characteristic distribution of galaxy masses and densities. What remains to be shown is whether the giant voids in the galaxy distribution, such as that in Bootes, and the enormous aggregates of galaxies, such as that in the Serpens-Virgo region some fifty million megaparsecs across, can be reconciled with such a theory. One of the most tantalizing aspects of the currently moribund idea of a universe dominated by massive neutrinos was that it naturally predicted the presence of large filaments and sheets of galaxies, separated by huge voids. It may not be so easy for a cold particle-dominated universe to meet the same challenge.

Joseph Silk is in the Astronomy Department, University of California, Berkeley, California 94720.

\title{
Astrophysics
}

\section{Oscillations of degenerate stars}

\section{from Arthur N. Cox}

RECENTLY there has been renewed interest in the oscillations of degenerate dwarf stars. Very-low-luminosity variations were first seen in the white dwarf HL Tau-76 by Landolt some 20 years ago ${ }^{1}$, and since then, luminosity variations with periods ranging from hundreds to thousands of seconds have been detected in many other white dwarfs. The past few months have seen several predictions confirmed by new observational discoveries, and the scope of our research and understanding of degenerate star oscillations greatly expanded.

The stars that have stimulated the interest are very small, with radii ranging from $1-10$ per cent of the radius of our Sun, only slightly bigger than that of the Earth, and a probable mass of 0.6 solar masses $\left(M_{0}\right)$. Their mean density is therefore very high, about $10^{6} \mathrm{~g} \mathrm{~cm}^{-3}$, implying the presence of electron degenerate matter throughout most of their mass. Because degenerate matter conducts heat easily, white dwarf stars have core temperatures as low as $10^{7} \mathrm{~K}$ or less and, thus, are much cooler than any other stars except the lower-mass very-faint red dwarfs. White dwarfs produce no nuclear energy because any potential fuels, such as hydrogen and helium, are found only in their cool surface layers, so their luminosity is low. Because of their very small size and low luminosity (less than 1 per cent of that of our Sun) it has taken billions of years for the surfaces of these stars to cool to their current temperatures, typically $10,000 \mathrm{~K}$.

Stellar evolution theory predicts that stars with masses of up to at least $6 \mathrm{M}$. evolve to become white dwarfs (see the figure). As these stars burn their initially copious supply of hydrogen to helium and the helium to carbon, oxygen and neon in a growing electron-degenerate core, they expand and increase in luminosity, to become red giants or supergiants. Whien they have reached a radius of perhaps 100 or more solar radii, they lose mass, sometimes to produce planetary nebula shells, by shedding all but the CNONe core resulting from the hydrogen and helium burning. The remaining core has a surface temperature typically of $150,000 \mathrm{~K}$ and a luminosity of 10,000 solar luminosities. Because the stars have lost the layers in which nuclear processes take place, they can no longer produce any new energy to sustain their luminosity and they rapidly (within no more than $10,000-100,000$ years) become very small and hot. Finally,

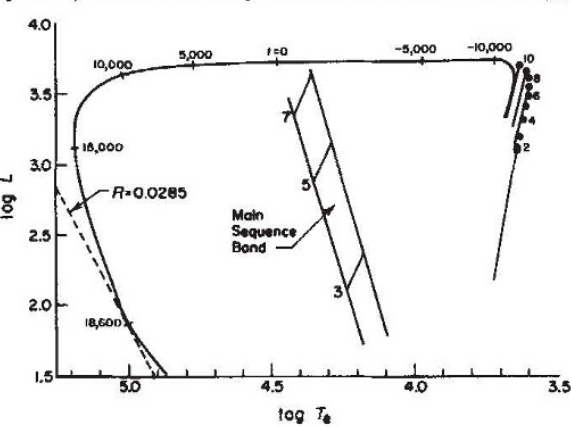

Hertzsprung-Russell diagram where luminosity is plotted versus the surface temperature, showing the evolution of a 0.6 $M$. star (adapted from ref.10). The initial increasing luminosity tracks for stars of 3,5 , and $7 \mathrm{M}$. are indicated as the main sequence band. The final stages of nuclear burning of the $0.6 \mathrm{M}$. star result in 10 helium shell flashes where the luminosity is greatly increased for durations of hundreds of years. These are marked at the very coolest surface temperatures where the mean emitted luminosity is still increasing with time. Zero time is arbitrarily set at the moment when the surface temperature increases to $30,000 \mathrm{~K}$. The track represents hot dwarfs (upper left), turning as they slowly cool to become very faint white dwarfs (below the bottom of the figure). The minimum radius of the hot dwarfs is 0.0285 solar radius, and later this radius decreases as the conventional white dwarf is formed.

slow cooling turns these hot dwarfs into faint white dwarfs after millions of years.

Many hot luminous stars have been discovered and very recently five of them have been found to show 5 - 30 minute period variations in their luminosity of a few per cent (ref. 2 and H.E. Bond \& A.D. Grauer, personal communication). These stars constitute a new class of variable, called PG variables, after the first studied PG1159-035 which is perhaps the hottest variable star in the sky. Oscillations of these stars are thought to result from the periodic ionization of carbon and oxygen in their surface layers. While they oscillate, the stars deviate from their spherical shape, hence their classification as nonradial pulsators. Last year, Starrfield et al. predicted that the stars would pulsate in nonradial $g$ modes only if about half of the mass of their outer pulsation-driving layers was oxygen, and helium comprised only a small fraction ${ }^{3}$. Now, a large oxygen abundance has been verified spectroscopically ${ }^{4}$. Observations of helium at the stars' surfaces, however, indicate incomplete loss of this fuel. If there is enough helium, another shell flash may occur during the stars' evolution. Further studies of the longer-period K1-16 planetary nebula central star listed in the Kohoutek catalogue are underway to see what pulsations would be predicted at earlier and cooler stages of this luminous stellar evolution.

The same cyclical ionization of hydrogen and helium that drives the pulsations of Cepheids also causes the much cooler and older ZZ Ceti stars, the classical white dwarfs, to pulsate. The $x$ and $\gamma$ effects drive motions by both blocking and hiding the radiative luminosity at key compressed phases of the pulsation cycle. At subsequent phases, the radiation is allowed to flow more strongly so that the oscillations are driven as the stars re-expand.

A further intriguing class of variable stars is the DB white dwarfs. These stars have helium-rich atmospheres and, probably, helium-rich pulsation-driving layers with temperatures of $100,000 \mathrm{~K}$ or more. They do not have hydrogen ionization driving, but they do have the driving mechanisms from the ionization of helium. Winget et al. predicted that these helium stars should pulsate in nonradial modes ${ }^{5}$, and recently this has been confirmed in three cases (ref.6 and H.E. Bond \& A.D. Grauer, personal communication).

What does the future hold in store? Several groups ${ }^{7-9}$ have predicted that radial pulsations in high overtones should occur in white dwarfs. Perhaps the next observational discovery will be that these approximately 1 -second oscillations also exist.

1. Landolt, A.U. Astrophys. J. 153, 151 (1968)

2. Bond, H.E. Grauer, A.D. Green, R.F. \& Liebert, J.W. Astrophys. J. 279, 751 (1984).

3. Starrfield, S.G., Cox, A.N., Hodson, S.W. \& Pesnell, W. D. Astrophys. J. Lett. 268, L27 (1983).

4. Sion, E.M., Liebert, J. \& Starrfield, S.G. Astrophys J. (in the press)

5. Winget, D.E., Van Horn, H.M., Tassoul, M., Hansen, C.J. \& Fontaine, G. Astrophys. J. Lett. 268, L33 (1983).

Winget, D.E. Robinson, E.L., Nather, R.E. \& Balachandran, S. Astrophys. J. Lett 279, L15 (1984).

7. Cox, A.N., Hodson, S.W. \& Starrfield, S.G. Lect. Notes Phys. 125, 458 (1980)

8. Saio, H., Winget, D.E. \& Robinson, E.L. Astrophys. J. 265, 982 (1983)

9. Starrfielc, S.G., Cox, A.N., Hodson, S.W. \& Clancy, S.P. Astrophys. J. 269, 645 (1983).

10. Iben, I. \& Renzini, A. A. Rev. Astron. Astrophys. 21, 271 (1983).

Arthur N. Cox is a Fellow of the Los Alamos National Laboratory, PO Box 1663, Los Alamos, New Mexico 87545. 